\title{
Messad / Castellum Dimmidi (Préhistoire)
}

\author{
C. Roubet et I. Amara
}

\section{OpenEdition}

Journals

Édition électronique

URL : http://journals.openedition.org/encyclopedieberbere/570

DOI : $10.4000 /$ encyclopedieberbere. 570

ISSN : 2262-7197

\section{Éditeur}

Peeters Publishers

\section{Édition imprimée}

Date de publication : 30 décembre 2010

Pagination : 4895-4898

ISBN : 978-90-429-2368-3

ISSN : 1015-7344

\section{Référence électronique}

C. Roubet et I. Amara, « Messad / Castellum Dimmidi (Préhistoire) », Encyclopédie berbère [En ligne], 31 | 2010, document M95a, mis en ligne le 08 octobre 2020, consulté le 12 octobre 2020. URL : http:// journals.openedition.org/encyclopedieberbere/570 ; DOI : https://doi.org/10.4000/ encyclopedieberbere. 570

Ce document a été généré automatiquement le 12 octobre 2020.

(c) Tous droits réservés 


\title{
Messad / Castellum Dimmidi (Préhistoire)
}

\author{
C. Roubet et I. Amara
}

1 La localité de Messad, au Sud-Est de Djelfa, offre plusieurs sites préhistoriques d'âge Holocène. Au Sud du djebel Boukahil (1363 m d'altitude), à $15 \mathrm{~km}$ au Nord-Est de Messad, on découvre de nombreux témoins d'habitats en plein air et des emplacements rocheux ornés de gravures. Le site antique, le Castellum Dimmidi servit d'avant poste militaire romain (Picard 1947 ; cf. notice suivante).

2 Les gisements de Ksar-Zaccar, Daïet Stel et d'Aïn Naga ont été signalés aux XIX-XX siècles par différents auteurs (Hatmayer 1882 ; Flamand 1914; Roffo 1935 ; Bellin 1954, Lethielleux 1965). Certains sites d'art rupestre ont été relevés et publiés par H. Lhote (1984).

3 A Aïn Naga, au pied d'une barre rocheuse de grés, D. Grébénart (1969) fit d'excellentes fouilles dans un dépôt en cours de déflation. Il découvrit en superposition deux ensembles culturels. L'un rattaché au Capsien* supérieur, l'autre au Néolithique* (?).

4 Le Capsien supérieur se trouvait conservé à l'intérieur d'une couche argilo-cendreuse gris clair, s'étalant sur une quarantaine de mètres, presque sans coquille d'Hélix; elle livra avec l'habituel mobilier lithique, de rares fragments d'os poli, une parure marine comprenant un dentale sectionné et une nassa gibbosula. L'ancienneté de ce niveau a été établie à partir de datations calibrées (Stuiver et Reimer 1993, note 1),

- sur charbon Gif $1220,9170 \pm 200$ BP soit : 9120-7754 cal BC,

- sur Hélix sans réf., $8900 \pm 280$ BP soit : 8781-7356 cal BC,

- sur Hélix sans réf., $9300 \pm 300$ BP soit : 9318-7676 cal BC.

Le niveau Néolithique :

- sur charbon, Gif 1221, $7500 \pm 220$ BP soit : 7022-5913 cal BC (note 2) se compose surtout de lamelles à bord abattu, de coches, de microlithes* géométriques, de microburins*: tous ces instruments maintiennent une forte tradition capsienne. Ce niveau est pourvu en tests d'œuf d'autruche, dont un gravé ; il comporte aussi de petits tessons décorés issus de vases modelés à large ouverture, ornés d'impressions flammées, ce décor 
suggère des influences sahariennes incontestables; on observe aussi l'arrivée d'armatures de flèches bifaciales, d'influence saharienne, la présence d'un racloir à retouche biface, mais pas encore de hache, ni de herminette en pierre polie. En somme, le substrat lithique reste capsien et son caractère accentué fit reconnaitre l'existence du Néolithique de tradition capsienne (Grébénart 1969, 1970 et Camps 1974, p. 312-314), selon l'acception de R. Vaufrey. Rappelons que C. Roubet a démontré que plus un contexte culturel était chargé d'éléments de tradition capsienne, moins forte, voire faible, était alors la Néolithisation (Roubet 1979). Faute de bonnes conditions de préservation, la faune consommée très mal conservée d'Aïn Naga ne contribua pas à préciser le statut économique de ce niveau, considéré comme prédateur.

7 Aucune autre recherche n'a été entreprise depuis 1970. Nous rattacherons donc un niveau comme D. Grébénart à un Capsien supérieur. L'autre peu modifié par l'arrivée manifeste mais faible de la Néolithisation, étant daté du VIII-VII ${ }^{\mathrm{e}}$ millénaire cal BC. Mais on ne peut pas en rester à ce constat incertain aujourd'hui. Mettons donc provisoirement en marge le contenu matériel de ce niveau et comme cela est proposé ci-dessous par C. Roubet, voyons aussi ce qu'apporte l'art des alentours.

8 L'art rupestre du site en renouvelle l'intérêt. Le thème du bélier* coiffé, bien connu dans l'Atlas saharien, exceptionnellement présent dans le site d'Ain Naga (Camps 1974, Pl. XXII, $n^{\circ} 1$ ), retient en particulier notre attention. Il appuie l'hypothèse d'une fréquentation soutenue de ce territoire, postérieurement aux datations citées. En effet, à l'intérieur de ces amas gréseux accueillants, ce sont sans doute de nouveaux pasteurs transhumants qui occupèrent ces aires de campement et de pâturage, pratiquant une domestication initiale des ovins. Pourquoi choisir de retenir cette hypothèse ? Puisant dans le bestiaire rupestre local, non encore daté, cette proposition prend en compte la présence de deux cas de gravures de béliers, isolés, signalées dans la région. Il s'agit d'œuvres soignées gravées et polies, sans repentir ni tâtonnement, présentant chaque fois un seul "bélier à sphéroïde ", souvent associé à un homme. Si, par hypothèse, on osait extraire ce thème de l'animal symbolique, domestique, gravé, du contexte d'animaux sauvages, on remarquerait alors qu'il étend vers l'est l'aire géographique de la thématique du bélier à l'Algérie orientale. Certes, sans pouvoir dater ici aussi son apparition, mais en ne l'envisageant pas non plus antérieure mais plutôt contemporaine de ce niveau de néolithique (?) initial. La fenêtre chronologique attribuable à cette domestication indirectement attestée par l'art de ce bélier d'Aïn Naga se situerait au plus tôt vers le VII è millénaire cal BC (sans consommation d'animaux domestiques). Ajoutons que cette gravure naturaliste si bien mise en évidence sur ces rochers par des graveurs compétents, paraît aussi être géographiquement la plus proche du massif de l'Aurès, occupé presque au même moment par des pasteurs (cf. note 2).

9 Ajoutons que, grâce à d'autres relevés précis de G. Lefebvre dans la région de Djelfa et au Kef Bou Bekker en particulier, c'est l'Ovis longipes Fitz. saharien qui a été identifié, symboliquement coiffé (Camps 1974, p. 328), et non le mouton à queue épaisse, qui se répandra plus tard en Algérie orientale.

10 En somme, l'information induite par cette gravure d'un bélier coiffé à Aïn Naga est décisive pour reconsidérer l'intérêt du site. S'articulant chronologiquement et 
archéologiquement bien avec les diverses données, passant du Capsien supérieur au Néolithique de tradition capsienne initial :

- Elle permet de donner de la durée et un devenir économique au contexte culturel très conservateur dit néolithique par D. Grébénart.

- Elle accrédite l'hypothèse d'une totale modification comportementale des groupes successifs venus s'installer dans la région; elle implique l'élevage d'ovins, la naissance de troupeaux, etc.

- Elle conduit à supposer l'inauguration d'un nouveau genre de vie, l'ouverture au monde et aux autres, un enrichissement en biens de consommation.

- Elle pérennise la pratique de l'échange.

11 L'attestent, dans le niveau qualifié de Néolithique : l'introduction de la céramique avec un décor flammé obtenu par impression pivotante (que l'on retrouve en région saharienne), et l'introduction d'armatures bifaciales à pédoncule (d'origine saharienne).

Le laisse entrevoir : la gravure du bélier coiffé d'Aïn Naga, montrant un mouton domestique adulte, docile, portant au cou un large lien décoré attestant la soumission ; cet animal à longs poils, membres longs, et profil convexe de la tête est précédé par un homme non armé.

Ainsi, la Néolithisation de la région de Messad, fondée sur l'élevage, pourrait-elle être considérée comme affirmée dès le VII-VIè millénaire (note 2), et pratiquée tant que les conditions climatiques restèrent favorables. Ce territoire s'ouvrant sur un Bas-Sahara propice aux communications, pourrait avoir assuré par le biais d'échanges, l'acculturation d'autres populations épipaléolithiques du piémont atlasique oriental et méridional.

\section{Notes}

1. Les datations ont été calibrées grâce au programme CALIB 5.0.1 (Stuiver-Reimer 1993), avec le module IntCal04, pour les échantillons terrestres.

2. Début du $\mathrm{VI}^{\mathrm{e}}$ et durant le $\mathrm{V}^{\grave{e}}$ millénaire, dans la grotte Capéletti du Khanguet Si Mohamed Tahar* de l'Aurès.

\section{BIBLIOGRAPHIE}

BELLIN P., 1954 - «L'art rupestre des Ouleds Naïl ». BSPF, t. LI, p. 205-206.

FLAMAND G.B.M., 1914 - « Deux stations nouvelles de pierres écrites (gravures rupestres)

découvertes dans le cercle de Djelfa, Sud-algérois (Algérie) ». L'Anthropologie, t. XXV, p. 433-458.

CAMPS G., 1974 - Les civilisations préhistoriques de l'Afrique du Nord. Doin, Paris.

GREBENART D., 1969 - « Aïn Naga : Capsien et Néolithique des environs de Messad ». Libyca, t. 17, p. 93-197.

GREBENART D., 1970 - « Problèmes du Néolithique près d'Ouled Djellal et de Djelfa : Botma Si

Mammar et Safiet Bou Rhenan ». Libyca, t. 18, p. 47-66. 
HARTMAYER Cpt., 1882 - « Notice sur les monuments préhistoriques du cercle de Djelfa ». Bulletin de correspondance africaine dans la région de Djelfa, fasc. 1, p. 118-124.

LETHIELLEUX J., 1965 - « La station de gravures rupestres de Daïet es-Stel ». Libyca, t. XV, p. 207-213. LHOTE H., 1984 - Les gravures rupestres de l'Atlas Saharien : monts des Ouled Nail et la région de Djelfa. Alger, Office du Parc national du Tassili, $291 \mathrm{p}$.

PICARD G.-Ch., 1947 - Castellum Dimmidi. Gouvernement général de l'Algérie. Direction des Antiquités. Mission archéologique.

ROFFO Dr. P., 1935 - « Découvertes préhistoriques dans la région de Djelfa ». Revue anthropologique, t. XLV, p. 47-59.

ROUBET C., 1979 - Economie pastorale pré-agricole en Algérie orientale. Le Néolithique de tradition capsienne. Exemple l'Aurès. CNRS, Antiquités Africaines.

STUIVER M., REIMER, P.J., 1993. "Extended 14C data base and revised CALIB 3.0 14C Age calibration program". Radiocarbon, 35, 1, p. 215-230.

\section{INDEX}

Mots-clés : Art, Art rupestre, Capsien, Néolithique, Préhistoire 